\title{
Monte Carlo-Free Prediction of Spurious Performance for ECDLL-Based Synthesizers
}

\author{
Amin Ojani, Behzad Mesgarzadeh and Atila Alvandpour
}

Linköping University Post Print

Tweet

N.B.: When citing this work, cite the original article.

Amin Ojani, Behzad Mesgarzadeh and Atila Alvandpour, Monte Carlo-Free Prediction of Spurious Performance for ECDLL-Based Synthesizers, 2015, IEEE Transactions on Circuits and Systems Part 1: Regular Papers, (62), 1, 273-282.

http://dx.doi.org/10.1109/TCSI.2014.2347231

(C2015 IEEE. Personal use of this material is permitted. However, permission to reprint/republish this material for advertising or promotional purposes or for creating new collective works for resale or redistribution to servers or lists, or to reuse any copyrighted component of this work in other works must be obtained from the IEEE.

Postprint available at: Linköping University Electronic Press http://urn.kb.se/resolve?urn=urn:nbn:se:liu:diva-110393 


\title{
Monte Carlo-Free Prediction of Spurious Performance for ECDLL-Based Synthesizers
}

\author{
Amin Ojani, Student Member, IEEE, Behzad Mesgarzadeh, Member, IEEE, and \\ Atila Alvandpour, Senior Member, IEEE
}

\begin{abstract}
Misalignment of delay-locked loop (DLL) output edges creates an undesired periodicity, resulting in reference harmonic tones at the output spectrum of edge-combining DLL (ECDLL)-based frequency synthesizers. These spurious tones corrupt the spectral purity to an unacceptable level for wireless applications. The spur magnitude is a random variable defined by the reference frequency, number of DLL phases, harmonic order, stage-delay standard deviation (SD), duty cycle distortion (DCD) of the reference clock, and static phase error (SPE) of the lockedloop due to charge pump/phase detector imperfections. Hence, to estimate the spurious performance of such synthesizers, exhaustive Monte Carlo (MC) simulations are inevitable. Based on closed-form expressions, this paper proposes a generic predictive model for harmonic spur characterization of ECDLLbased frequency synthesizers, whose prediction accuracy is independent of synthesizer design parameters and system nonidealities. Therefore, it can replace MC method to significantly accelerate the iterative design procedure of the synthesizer, while providing comparable predictions in terms of robustness and accuracy to that of MC. Validity, accuracy, and robustness of the proposed prediction method against wide-range values of nonidealities are verified through MC simulations of both the behavioral model and transistor-level model of the synthesizer in a standard 65-nm CMOS technology.
\end{abstract}

Index Terms - delay mismatch, DLL, duty cycle distortion, frequency synthesizer, harmonic spur, Monte Carlo, periodic jitter, predictive model, static phase error

\section{INTRODUCTION}

SPeCtral purity is a key requirement for frequency generation schemes in wireless applications. In integer locked-loop-based frequency synthesis, where the system is locked to a low-frequency reference clock, non-idealities of the system can produce an erroneous periodicity, known as periodic jitter, resulting in appearance of reference harmonic tones at the spectrum of the synthesized carrier. These harmonic spurs can potentially downconvert out-of-band interferers into the desired band, corrupting the wanted signal. Delay-locked loop (DLL)-based frequency synthesis achieves low close-in phase noise by taking advantage of the lowaccumulative-jitter property of DLLs, which is obtained by periodically resetting the jitter back to zero, and consists of two main approaches. In recirculating DLL-based approach [1]-[7] where the multiplied clock is generated by a similar mechanism as in ring voltage-controlled-oscillator (VCO)based phase-locked loops (PLL), the harmonic spur-to-carrier ratio (SCR) is directly defined by the output frequency and static phase error (SPE) of the locked-loop [5], [7]. Edgecombining DLL (ECDLL)-based synthesizers [8]-[16], on the other hand, are more prone to generation of spurious tones. As the carrier is produced by combining the equally-spaced DLL output edges, any sort of non-ideality, including SPE, duty cycle distortion (DCD), and stage-delay mismatch (as a stochastic variable), will cause misalignment in those edges, degrading the spurious performance. Consequently, the SCR in ECDLLs becomes a random variable which is defined by the design parameters, i.e., the reference frequency and number of DLL phases, as well as the system non-idealities, i.e., SPE, DCD, and stage-delay standard deviation (SD) [16]. As a result, spur characterization of ECDLL-based synthesizers requires exhaustive transient Monte Carlo (MC) simulations which significantly slow down their design procedure.

Among those prior arts [10], [12], [13], [16] which study the spurious characteristics of ECDLL synthesizers, a comprehensive behavioral model is proposed in [16] which includes all the aforementioned sources of harmonic spurs in such synthesizers, and provides an analysis which leads to derivation of a closed-form approximation for synthesizer SCR. However, there are two limitations regarding the approximation in [16]. First, the prediction accuracy is guaranteed only for some bounded values of SPE, DCD, and delay SD, and will degrade otherwise. Second, due to dependency of the prediction error upon the synthesizer design parameters and system non-idealities, it is challenging to define and formulate generic accuracy bounds of the model, without performing case-specific MC simulations for a given design scenario. These limitations indicate the necessity and importance of developing a more generic and accurate predictive model, which provides predictions that are as robust as those achieved using statistical simulations, and hence, can be employed as a general replacement to MC method.

In this work, the main objective is to mitigate those major limitations of the approximation model in [16], by developing generic and robust predictions which can thoroughly replace exhaustive MC method for spur characterization of ECDLL synthesizers. In order to do so, the paper first investigates the behavior of even- and odd-order harmonic spurs at the output spectrum of such synthesizers, and demonstrates that depending on the relations among SPE, DCD, and delay SD values, either of those harmonics can have the largest spur level. This implies that the SCR needs to be accurately characterized and predicted for both the adjacent and alternate harmonics to the carrier. Afterwards, the limitations regarding the approximation model in [16] are elaborated, emphasizing the need for more accurate predictions. Accordingly, based on closed-form expressions, the paper proposes a generic predictive model for ECDLL synthesizers, whose accuracy in 
SCR estimation is independent of the design parameters or system non-idealities. This model exhibits comparable accuracy and robustness with that of the MC method, whereas it eliminates the extensive simulation time which is involved in statistical MC simulations. Hence, the proposed model can reliably replace $\mathrm{MC}$ method to accelerate the iterative design procedure of ECDLL-based frequency synthesizers.

The paper is organized as follows. Section II provides the necessary background regarding the behavioral model of ECDLL-based frequency synthesizers. In Section III, dissimilar characteristics of even- and odd-order harmonic spurs are investigated. Section IV demonstrates the limitations of the model in [16] for SCR estimation, indicating the demand for a more generic predictive model for SCR characterization, which is then proposed in Section V. In Section VI, the proposed model is validated through MC simulations of the behavioral model as well as the transistorlevel model of ECDLL synthesizer in a standard 65-nm CMOS process. Finally, the paper is concluded in Section VII.

\section{BACKGROUND TO ECDLL BEHAVIORAL MODEL}

Assume that the charge pump (CP) ECDLL synthesizer shown in Fig. 1 employs a voltage-controlled delay line (VCDL) of $N$ delay stages and locked to the rising edge of a reference clock of frequency $f_{\text {ref. }}$. The corresponding ECDLL model which includes SPE, DCD, and delay mismatch, is illustrated as a timing diagram in Fig. 2. The synthesized output is generated by summation of the rising and falling DLL output current edges ( $N$ is odd) [8] and contains a fundamental tone at $f_{c}=N \times f_{\text {ref }}$ and reference harmonic spurs at $f_{s}=n \times f_{\text {ref }}$, where $n$ is an integer harmonic order. It can be shown that the mismatches of the rising- and falling-edge delays are modeled as two independent Gaussian random variables [13], [16]-[18],

$$
\Delta t_{d, r} \sim \mathrm{N}\left(\mu_{r}, \sigma_{r}^{2}\right), \Delta t_{d, f} \sim \mathrm{N}\left(\mu_{f}, \sigma_{f}^{2}\right)
$$

where $\mu_{r}, \sigma_{r}^{2}, \mu_{f}$, and $\sigma_{f}^{2}$ are the mean and variance of risingand falling-edge delays, respectively. In Fig. 2, $k$ models the input clock pulsewidth. So, the time-domain DCD value $T_{d c d}$ is

$$
T_{d c d}=\left(T_{r e f} / 2\right)-k
$$

Due to phase detector (PD)/CP mismatches, the lock period $T_{\text {lock }}$ deviates from $T_{\text {ref }}$ with a time-domain SPE of $T_{\text {spe }}$. Hence,

$$
T_{\text {lock }}=T_{\text {ref }}+T_{s p e} .
$$

If the mean values of rising- and falling-edge delays are equal, i.e., $\mu=\mu_{r}=\mu_{f}=T_{\text {lock }} / N$, then $\Delta t_{d, r}$ and $\Delta t_{d, f}$ are represented as

$$
\Delta t_{d, r m}=\frac{T_{\text {lock }}}{N}-t_{\text {avg }}+G_{r m}, \Delta t_{d, f m}=\frac{T_{\text {lock }}}{N}-t_{\text {avg }}+G_{f m}
$$

where $m$ is the stage index, $m \in[1, N]$, and $G_{r m}$ and $G_{f m}$ are two independent sets of zero-mean Gaussian variables defined as

$$
G_{r 1}, \ldots, G_{r N} \sim \mathrm{N}\left(0, \sigma_{r}^{2}\right), G_{f 1}, \ldots, G_{f N} \sim \mathrm{N}\left(0, \sigma_{f}^{2}\right) .
$$

Furthermore, $t_{\text {avg }}$ is the averaged sum of $N$ random variables $G_{r m}$, i.e., the rising-edge delay mismatches. It resembles the mismatch-averaging property of the loop and can be derived as

$$
t_{\text {avg }}=\frac{1}{N} \sum_{m=1}^{N} G_{r m} \sim \mathrm{N}\left(0, \sigma_{r}^{2} / N\right) .
$$

Analyzing Fourier series of the DLL phases in Fig. 2, $a_{n}$ and $b_{n}$

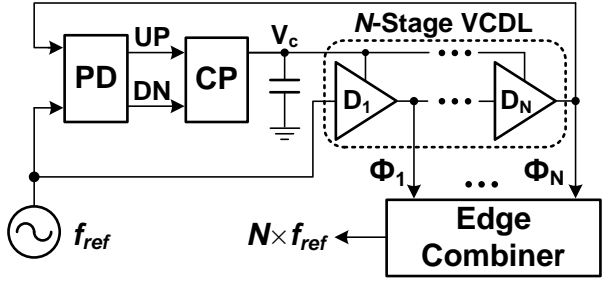

Fig. 1. Block diagram of an analog ECDLL-based frequency synthesizer.

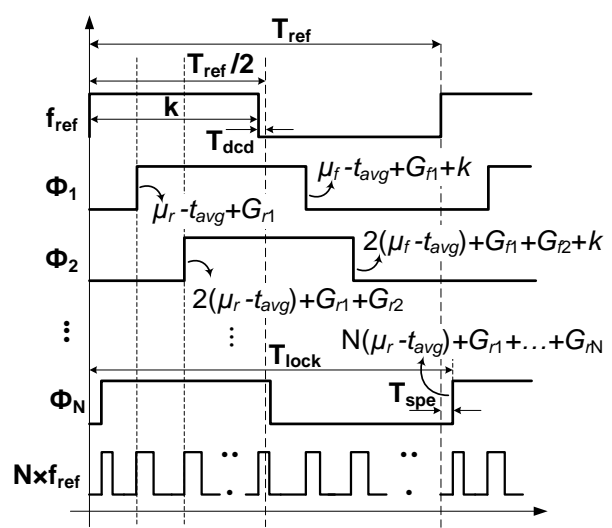

Fig. 2. Timing diagram of ECDLL, modeling SPE, DCD, and delay mismatch.

Fourier coefficients of the synthesized output are found [16] as

$$
\begin{aligned}
& a_{n}=\frac{2 A_{0}}{T_{\text {ref }}} \sum_{m=1}^{N}\left(\int_{m \mu+\sum_{i=1}^{m} G_{r_{r}}-m t_{a v g}}^{k+m \mu+\sum_{\text {arg }}^{m} G_{f}-m t_{\text {vig }}} \cos \left(\frac{2 n \pi}{T_{\text {ref }}} t\right) d t\right)
\end{aligned}
$$

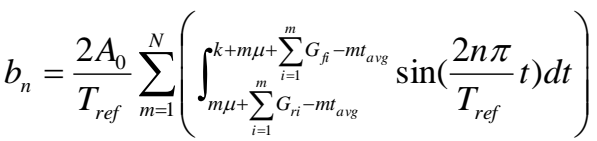

where $A_{0}$ and $T_{r e f}$ are the amplitude and period of the reference clock, respectively. Note that (7) and (8) correspond to the output Fourier coefficients of a current-summation edgecombiner (EC) [8]. Nonetheless, the presented behavioral model can be generally employed to find the Fourier coefficients of other EC types. Using (7) and (8), the analytical model of SCR for a given harmonic $n$ is expressed as

$$
S C R_{n}=S_{n} / C=\sqrt{a_{n}^{2}+b_{n}^{2}} / \sqrt{{a_{N}{ }^{2}+b_{N}{ }^{2}}^{2}}
$$

where $S_{n}$ and $C$ are the magnitudes of harmonic spur and carrier, respectively. Random variable SCR in (9) formulates the spurious performance of ECDLL synthesizers by modeling the effects of delay mismatch among the delay stages, SPE of the loop due to mismatch of up and down signals in $\mathrm{PD} / \mathrm{CP}$, and DCD of the reference clock. Equation (9) is utilized as a reference analytical model for evaluating the validity and accuracy of the prediction models throughout the paper.

\section{EVEN-ORDER VERSUS ODD-ORDER HARMONIC SPURS}

In this section, the spurious characteristics of the adjacent (even-order) and alternate (odd-order) harmonics at the output spectrum of ECDLL synthesizers are investigated. The purpose is to demonstrate that depending on the relations among SPE, DCD, and delay SD values, either of the adjacent or alternate harmonics to the carrier may have the largest spur level. This implies that the spurious performance requirement for a certain wireless standard needs to be verified through 


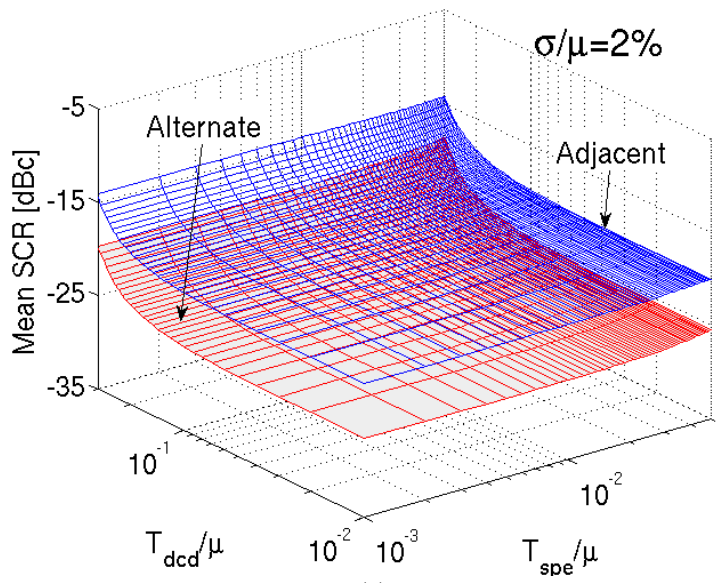

(a)

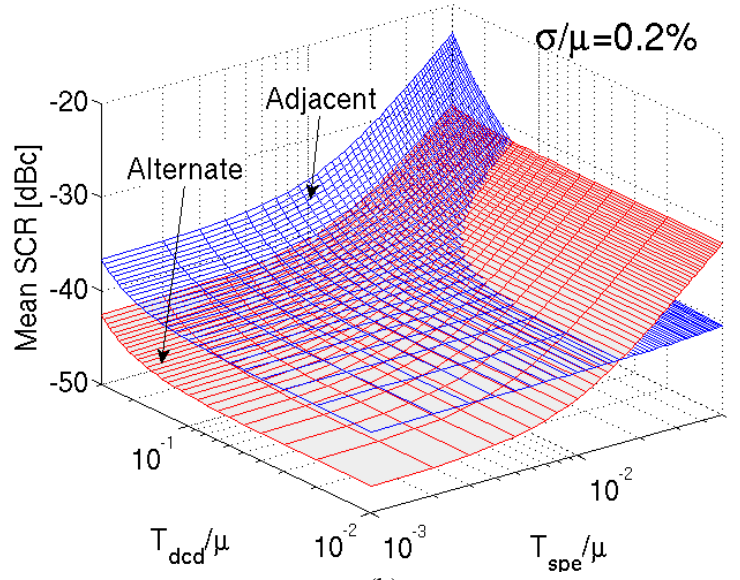

(b)

Fig. 3. MC simulation of the analytical mean SCR of (9) for the adjacent $\left(n=N-1, f_{s}=f_{c}-f_{\text {ref }}\right)$ and alternate $\left(n=N-2, f_{s}=f_{c}-2 f_{\text {ref }}\right)$ harmonics as a function of normalized DCD and SPE, using normalized delay SD of (a) $2 \%$ and (b) $0.2 \%$.

spur analysis of both the adjacent and alternate harmonics. Note that because in the utilized ECDLL model presented in the previous section, $N$ is assumed to be odd, the adjacent harmonics with $n=N \pm 1$ are of even orders.

For the presented analysis throughout the paper, an ECDLL synthesizer is utilized that is locked to a reference clock of $f_{\text {ref }}$ $=400 \mathrm{MHz}$ and employs a VCDL with $N=25$ stages, producing a carrier frequency of $f_{c}=10 \mathrm{GHz}$. Furthermore, DCD and SPE values are swept from $T_{d c d}=1$ to $30 \mathrm{ps}$ and $T_{\text {spe }}$ $=0.1$ to $5 \mathrm{ps}$, respectively. In addition, by choosing two normalized delay SD values of $\sigma_{r} / \mu_{r}=\sigma_{f} / \mu_{f}=\sigma / \mu=0.2 \%$ and $2 \%$, the synthesizer SCR is simulated for the adjacent $\left(f_{s}=f_{c}-\right.$ $\left.f_{\text {ref }}\right)$ and alternate $\left(f_{s}=f_{c}-2 f_{\text {ref }}\right)$ harmonics. Thus, four different test scenarios are investigated. Note that the values of nonidealities are selected in such a way that realistic corner cases regarding ECDLL synthesizer implementations are covered.

$\mathrm{MC}$ simulations of the analytical model of the synthesizer given in (9) are performed in MATLAB and the mean SCR results are plotted in Fig. 3. The $\mathrm{X}$ and $\mathrm{Y}$ axes correspond to the normalized SPE and DCD values, respectively. It can be observed from Fig. 3(a) that with a large delay SD of 2\% and within the utilized sweep range for SPE and DCD, the SCR of the adjacent harmonic always dominates that of the alternate one. However, it is not the case if smaller values of delay SD are utilized to achieve lower SCR which is required for wireless applications. As shown in Fig. 3(b), for a delay SD of $0.2 \%$ (an order of magnitude smaller than the previous case), as SPE increases, the SCR of the alternate harmonic can dominate. This observation indicates that there are dissimilar spurious characteristics for the even- and odd-order harmonics. However, as illustrated in Fig. 3(a), this different behavior is not revealed if SPE and DCD values are absorbed by a large stage-delay mismatch. From Fig. 3(b) it can be perceived that the mean SCR of the adjacent harmonic depends upon both the SPE and DCD values. On the other hand, for the alternate harmonic, the mean SCR is mainly defined by SPE value, and degrades significantly as SPE grows, regardless of DCD value. This behavior can be explained by evaluating the phase misalignment patterns on the transient waveform of the ECDLL output. Each non-ideality parameter (SPE, DCD, or delay SD) generates a unique pattern which results in certain harmonic behavior.

Distinctive characteristics of adjacent and alternate harmonic spurs in ECDLL synthesizers imply that to ensure a certain spurious performance, the SCR of both the harmonic spurs needs to be maintained below the required value. As a consequence, a generic predictive model should be able to accurately estimate SCR, not only for wide-range values of non-idealities, but also for both even and odd harmonic orders.

\section{LIMITATIONS OF THE APPROXIMATE MODEL}

The limitations regarding the approximation model of [16] are addressed in this section by evaluating its accuracy in SCR estimation in four different test scenarios, similar to those of the previous section. Demonstrating that the model can accurately predict the spurious performance only when the SPE and DCD values are sufficiently absorbed by the value of the delay SD, its limitations for being employed as a general replacement to MC method for spur characterization of ECDLL-based synthesizers are clarified.

Assuming that the harmonic Fourier coefficients $a_{n}$ and $b_{n}$ of the synthesizer output, expressed by (7) and (8) respectively, are two independently and identically distributed (iid) random variables, they are approximated in [16] as two zero-mean Gaussian variables with equal variances, i.e.,

$$
a_{n} \sim \mathrm{N}\left(0, \sigma_{c o f}^{2}\right), \quad b_{n} \sim \mathrm{N}\left(0, \sigma_{c o f}^{2}\right) \quad n \neq N
$$

and hence, the spur magnitude $S_{n}=\sqrt{a_{n}^{2}+b_{n}^{2}}$ is modeled as a Rayleigh random variable [19], whose mean value is

$$
\mu_{S_{n}}=\sigma_{c o f} \sqrt{\pi / 2}
$$

To examine the model accuracy, the simulated and predicted mean SCR values, from the analytical model (9) and the closed-form approximation model of [16], are compared over wide-range values of SPE and DCD. The results are plotted in Fig. 4 and 5 respectively, for a large and small normalized delay SD of $2 \%$ and $0.2 \%$. The transparent planes correspond to the MC-simulated SCR and the solid planes depict the predicted results. When delay SD is $2 \%$, the prediction follows the simulation quite closely for both the adjacent and alternate harmonic spurs shown in Fig. 4(a) and 4(b) respectively. Therefore, for the first two scenarios, a quite acceptable accuracy is provided. However, the achieved mean SCR values 


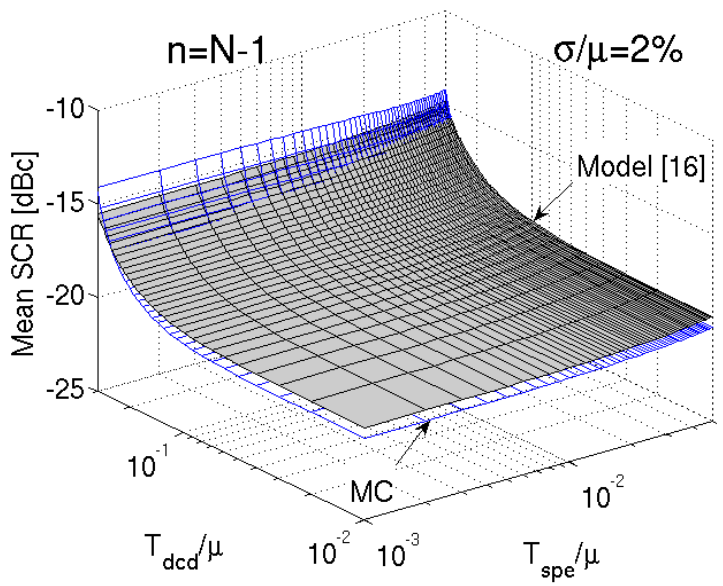

(a)

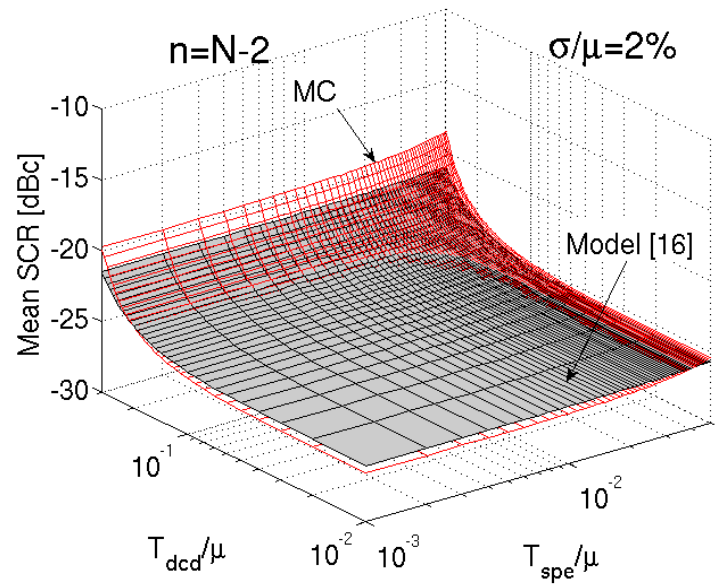

(b)

Fig. 4. MC simulation of the analytical model (9) versus the approximate prediction model of [16] for mean SCR values as a function of normalized SPE and $\mathrm{DCD}$, using normalized delay SD of $2 \%$ : (a) the adjacent, and (b) the alternate harmonic.

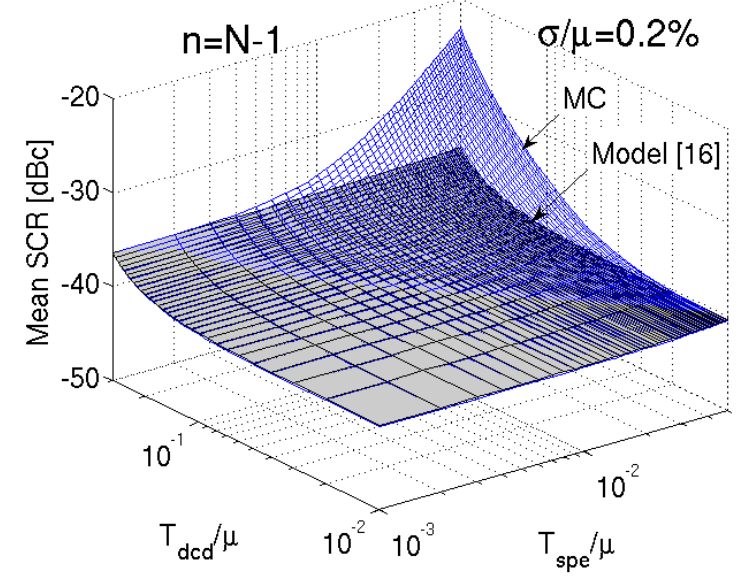

(a)

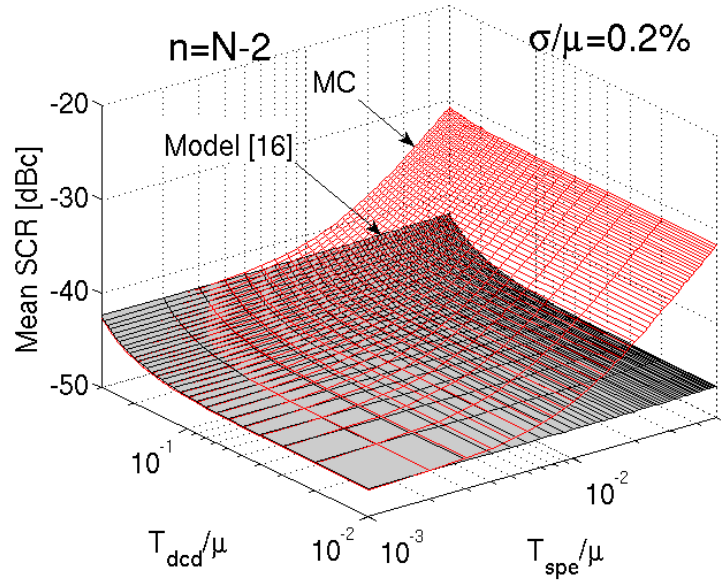

(b)

Fig. 5. MC simulation of the analytical model (9) versus the approximate prediction model of [16] for mean SCR values as a function of normalized SPE and DCD, using normalized delay SD of $0.2 \%$ : (a) the adjacent, and (b) the alternate harmonic.

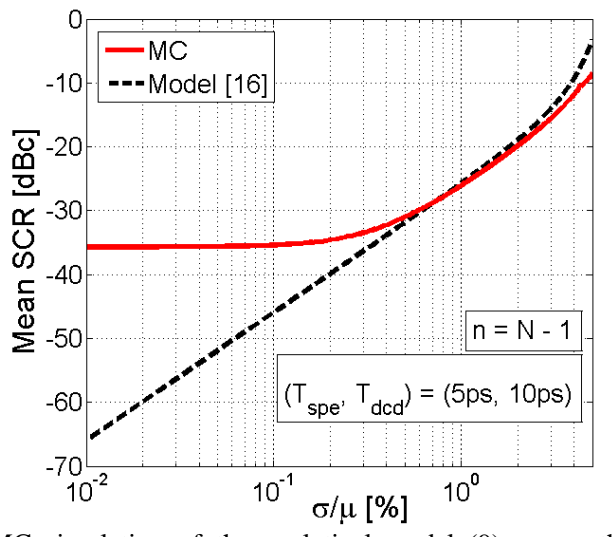

Fig. 6. MC simulation of the analytical model (9) versus the prediction model of [16] for mean SCR values as a function of normalized delay SD.

$(\sim-20 \mathrm{dBc})$ using the delay SD of $2 \%$ is not adequate for wireless applications. Hence, similar evaluations for the next two scenarios are repeated using a more practical delay SD of $0.2 \%$ and the results are plotted in Fig. 5. It can be observed that the accuracy of the prediction is largely degraded for both the adjacent and alternate harmonics, though with different error characteristics. As shown in Fig. 5(a), the prediction accuracy for the adjacent harmonic spur depends on both the SPE and DCD values, whereas for the alternate harmonic illustrated in Fig. 5(b), the accuracy is mainly dependent on the value of SPE. As it can be seen from Fig. 5, for a very small non-ideality pair of $T_{s p e}=1 \mathrm{ps}\left(T_{s p e} / \mu=10^{-2}\right)$ and $T_{d c d}=$ $10 \mathrm{ps}\left(T_{d c d} / \mu=10^{-1}\right.$, duty cycle $\left.=49.6 \%\right)$, the prediction error is 0 and $3 \mathrm{~dB}$, for the adjacent and alternate harmonics, respectively. However, since the adjacent spur is larger than the alternate one in this case, the corresponding $3-\mathrm{dB}$ error is not important. On the other hand, for a larger non-ideality pair of $T_{s p e}=5 \mathrm{ps}$ and $T_{d c d}=10 \mathrm{ps}$, the situation is quite different. The prediction error becomes as high as $5 \mathrm{~dB}$ and $15 \mathrm{~dB}$ for the adjacent and alternate spurs, respectively. Also, in contrast to the previous test case, the alternate spur is larger than the adjacent one. Note that these errors will be magnified if the delay SD is further reduced. This can be noticed from Fig. 6 which illustrates the mean SCR of the adjacent harmonic with respect to normalized delay SD and for a constant SPE-DCD pair of (5ps, 10ps). The MC simulation results represented by the solid line shows that for small delay SDs the mean SCR is mainly defined by SPE and DCD and not improved by further decreasing the delay SD. However, the prediction results of the model [16] (dashed line) cannot follow that of the MC.

Besides its conditional accuracy, there is another limiting factor concerning this model. It can be concluded by comparing Fig. 4 and 5 that the accuracy conditions are defined by the relations among the values of delay SD, SPE, and DCD. This makes it complicated to define those conditions and bounds in such a generic form which can be utilized for every design scenario without needing to perform 


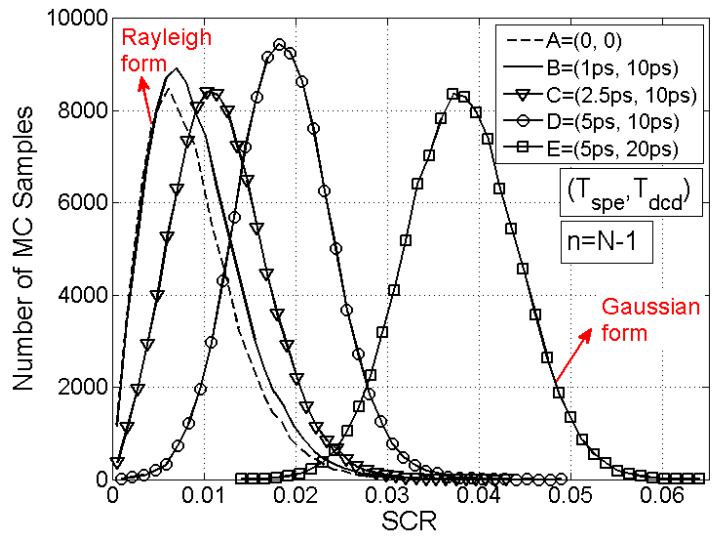

(a)

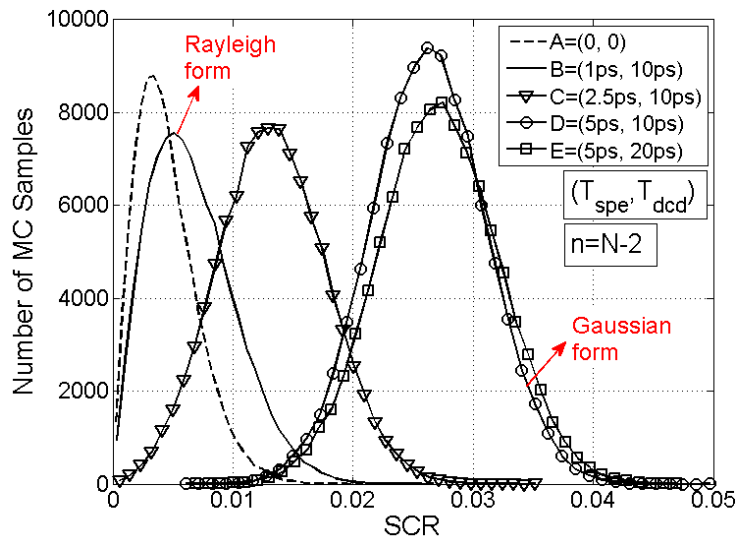

(b)

Fig. 7. SCR random variable identification: MC histogram of the analytical model (9), using a delay SD of $0.2 \%$ and different $\left(T_{s p e}, T_{d c d}\right)$ pairs: (a) the adjacent, and (b) the alternate harmonic.

case-specific simulations. This indicates that it is of great importance to develop a more generic predictive model for SCR estimation in ECDLL synthesizers, whose accuracy is independent from the design parameters and non-idealities.

\section{A GenERIC PREDICTIVE Model}

Different approaches can be employed to find the probability density function (PDF) of the SCR random variable defined in (9). An exact solution would be to derive the PDF of Fourier coefficients directly from the random nonidealities and then, by transformation of the resulting PDFs into their root sum square, calculate the PDF of SCR. By solving (7) and (8), and applying Taylor series approximation, expressions for $a_{n}$ and $b_{n}$ are derived as

$$
\begin{gathered}
a_{n}=\frac{2 A_{0}}{n \pi} \sum_{m=1}^{N}\left\{\alpha_{m} \kappa_{s}-\alpha_{m} \kappa_{s} \lambda^{2}\left(X_{f m}^{2}+X_{r m}^{2}\right)+\alpha_{m} \kappa_{c} \lambda\left(X_{f m}-X_{r m}\right)\right. \\
\left.-\beta_{m} \kappa_{s} \lambda\left(X_{f m}+X_{r m}\right)-\beta_{m} \kappa_{c} \lambda^{2}\left(X_{f m}^{2}-X_{r m}^{2}\right)\right\} \\
b_{n}=\frac{2 A_{0}}{n \pi} \sum_{m=1}^{N}\left\{\beta_{m} \kappa_{s}-\beta_{m} \kappa_{s} \lambda^{2}\left(X_{f m}^{2}+X_{r m}^{2}\right)+\beta_{m} \kappa_{c} \lambda\left(X_{f m}-X_{r m}\right)\right. \\
\left.+\alpha_{m} \kappa_{s} \lambda\left(X_{f m}+X_{r m}\right)+\alpha_{m} \kappa_{c} \lambda^{2}\left(X_{f m}^{2}-X_{r m}^{2}\right)\right\}
\end{gathered}
$$

where

$$
\begin{gathered}
X_{f m}=-\frac{m}{N} \sum_{i=1}^{N} G_{r i}+\sum_{i=1}^{m} G_{f i}, X_{r m}=-\frac{m}{N} \sum_{i=1}^{N} G_{r i}+\sum_{i=1}^{m} G_{r i} . \\
\lambda=\frac{n \pi}{T_{r e f}}, \kappa_{s}=\sin \frac{k n \pi}{T_{r e f}}, \text { and } \kappa_{c}=\cos \frac{k n \pi}{T_{r e f}} \\
\alpha_{m}=\cos \left(\frac{n \pi}{T_{r e f}}\left[\frac{2 m}{N}\left(T_{r e f}+T_{s p e}\right)+\frac{T_{r e f}}{2}-T_{d c d}\right]\right) \\
\beta_{m}=\sin \left(\frac{n \pi}{T_{r e f}}\left[\frac{2 m}{N}\left(T_{r e f}+T_{s p e}\right)+\frac{T_{r e f}}{2}-T_{d c d}\right]\right) .
\end{gathered}
$$

Deriving PDFs of $a_{n}$ and $b_{n}$ requires calculation of PDFs for the elements of (12) and (13), which are in form of time series and would result in complex analysis. In order to avoid dealing with complicated derivations involved in the direct PDF transformation method, another approach is employed in this work. The idea is to associate the SCR random variable to a well-known distribution so as to efficiently characterize the mean and PDF of the synthesizer SCR, based on the properties of that recognized distribution. In order to do so, an observation is made on different MC histograms of the SCR first. Then, the criteria regarding the identified distribution are inspected for the output Fourier coefficients of the synthesizer, and finally, the model parameters are determined from (12) and (13), using moments methods.

\section{A. Random Variable Identification}

To identify the distribution regarding the magnitudes of the harmonic spurs, MC simulations are performed on synthesizer's analytical model of (9), and the resulting SCR histograms are plotted in Fig. 7. For this purpose, a small (and more practical) delay SD of $0.2 \%$ is utilized to better observe the effect of SPE and DCD. Also, five different pairs of ( $T_{s p e}$, $\left.T_{d c d}\right)$ are employed for the simulations, i.e., $\mathrm{A}=(0,0), \mathrm{B}=$ (1ps, 10ps), $\mathrm{C}=(2.5 \mathrm{ps}, 10 \mathrm{ps}), \mathrm{D}=(5 \mathrm{ps}, 10 \mathrm{ps})$, and $\mathrm{E}=(5 \mathrm{ps}$, 20ps). By evaluating the plotted distributions in Fig. 7, it can be noted that the SCR exhibits the distribution of Rayleigh random variables for smaller SPE-DCD values, i.e., A and B pairs. Moving towards the largest input pair E, the distribution demonstrates a smooth transition from Rayleigh to Gaussian form. The observed behavior in the simulated distributions is in fact similar to that of Ricean random variables [19]. From the definition of such variables, the spur magnitude $S_{n}=\sqrt{a_{n}^{2}+b_{n}^{2}}$ will have a Ricean distribution if the harmonic Fourier coefficients $a_{n}$ and $b_{n}$ represented by (7) and (8), are two normal random variables with equal variances which are expressed as

$$
a_{n} \sim \mathrm{N}\left(\mu_{a_{n}}, \sigma_{c o f}^{2}\right), \quad b_{n} \sim \mathrm{N}\left(\mu_{b_{n}}, \sigma_{c o f}^{2}\right) \quad n \neq N
$$

where $\mu_{a n}$ and $\mu_{b n}$ are the mean values of $a_{n}$ and $b_{n}$ respectively, and $\sigma_{c o f}^{2}$ is their variance. Equation (18) should now be investigated by evaluating the variance and mean of the harmonic Fourier coefficients. The variances of $a_{n}$ and $b_{n}$ are plotted in Fig. 8(a) for the adjacent harmonic spurs as a function of SPE and DCD values. It can be observed that $a_{n}$ and $b_{n}$ variances maintain within a small range and almost close to each other, with a worst-case difference of around $2.5 \times 10^{-5}$. Fig. 8(b) shows that the mean values of $a_{n}$ and $b_{n}$ are zero for sufficiently small SPE and DCD values. However, for greater values of SPE and DCD, the mean values tend to deviate largely from zero, dissatisfying criteria (10). This, in fact, explains why for non-small SPE and DCD values, the harmonic spur levels cannot be predicted accurately with the approximation model of [16]. Note that the normality 


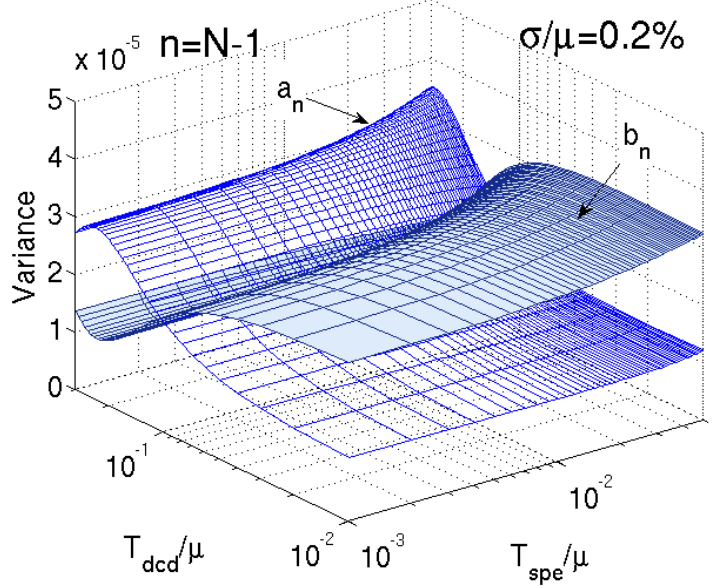

(a)

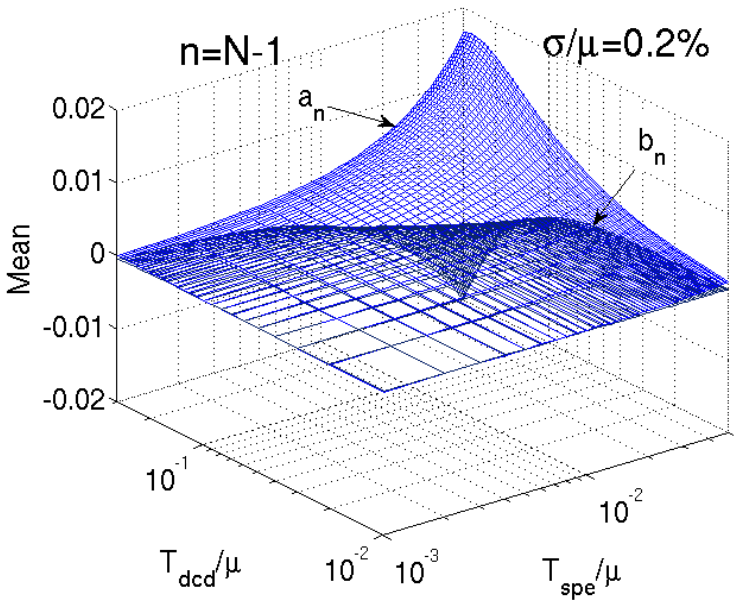

(b)

Fig. 8. MC simulation regarding (a) the variance, and (b) the mean of the adjacent harmonic Fourier coefficients $(n=N-1)$ as a function of normalized SPE and DCD, using normalized delay SD of $0.2 \%$.

condition of $a_{n}$ and $b_{n}$ in (18) can be verified using graphical tests [16]. Therefore, the harmonic spur magnitudes $S_{n}$ are modeled as Ricean random variables, with the following PDF and mean expressions [19].

$$
\begin{gathered}
p_{Z}(z)=\frac{z}{\sigma_{c o f}^{2}} \exp \left(-\frac{z^{2}+h^{2}}{2 \sigma_{c o f}^{2}}\right) I_{0}\left(\frac{h z}{\sigma_{c o f}^{2}}\right) \quad z \geq 0 \\
E\left[S_{n}\right]=\sigma_{c o f} \sqrt{\frac{\pi}{2}} \times e^{\frac{-K}{2}} \times\left[(1+K) I_{0}\left(\frac{K}{2}\right)+K I_{1}\left(\frac{K}{2}\right)\right]
\end{gathered}
$$

where $h$ is the magnitude of the vector $\left(\mu_{a n}, \mu_{b n}\right)$, i.e.,

$$
h=\sqrt{\mu_{a_{n}}^{2}+\mu_{b_{n}}^{2}}
$$

and $K$ is the Rice factor which is defined as

$$
K=h^{2} / 2 \sigma_{\text {cof }}^{2} \text {. }
$$

Also, $I_{0}(x)$ and $I_{1}(x)$ are the modified Bessel functions of the first kind with order zero and one, respectively, and defined as

$$
\begin{gathered}
I_{0}(x)=\frac{1}{\pi} \int_{0}^{\pi} e^{x \cos \theta} d \theta \\
I_{1}(x)=\frac{1}{\pi} \int_{0}^{\pi} e^{x \cos \theta} \cos (\theta) d \theta .
\end{gathered}
$$

\section{B. Determining Model Parameters}

After identifying the harmonic spur magnitudes in ECDLL synthesizers as Ricean random variables, the mean and PDF of $S_{n}$ in (19) and (20), need to be determined by calculating the values of $h$ and $\sigma_{c o f}$. Finding the first and second moments of (12) and (13), the mean and variance of $a_{n}$ and $b_{n}$ are determined [16] as

$$
\begin{array}{r}
\mu_{a_{n}}=E\left[a_{n}\right]=\frac{2 A_{0}}{n \pi} \sum_{m=1}^{N}\left\{\alpha_{m} \kappa_{s}-\alpha_{m} \kappa_{s} \lambda^{2} m\left(\sigma_{f}^{2}+\sigma_{r}^{2}\right)\right. \\
\left.-\beta_{m} \kappa_{c} \lambda^{2} m\left(\sigma_{f}^{2}+\sigma_{r}^{2}\left(\frac{2 m}{N}-1\right)\right)\right\} \\
\mu_{b_{n}}=E\left[b_{n}\right]=\frac{2 A_{0}}{n \pi} \sum_{m=1}^{N}\left\{\beta_{m} \kappa_{s}-\beta_{m} \kappa_{s} \lambda^{2} m\left(\sigma_{f}^{2}+\sigma_{r}^{2}\right)\right. \\
\left.+\alpha_{m} \kappa_{c} \lambda^{2} m\left(\sigma_{f}^{2}+\sigma_{r}^{2}\left(\frac{2 m}{N}-1\right)\right)\right\}
\end{array}
$$

$$
\begin{aligned}
\sigma_{c o f}^{2} \approx \frac{\operatorname{var}\left[a_{n}\right]+\operatorname{var}\left[b_{n}\right]}{2}=\left(\frac{2 A_{0}}{T_{r e f}}\right)^{2}\left(\sigma_{f}^{2}+\sigma_{r}^{2}\right) \\
\times\left(\frac{N(N+1)}{4}+\sum_{m=1}^{N-1} m\left(\alpha_{m} \sum_{i=m+1}^{N} \alpha_{i}+\beta_{m} \sum_{i=m+1}^{N} \beta_{i}\right)\right)
\end{aligned}
$$

The value of $h$ can be found by substitution of the numerical values of (25) and (26) into (21).

Now, knowing the Ricean random variable $S_{n}$, the ratio random variable $S C R_{n}$ defined in (9) should be determined. As discussed in [16], it can be shown that the random variable $C$ which represents the carrier magnitude, can be approximated by its mean value $E[C]$, and hence, (9) is simplified to $S C R_{n}=$ $S_{n} / E[C]$. This implies that the harmonic Fourier coefficients of $S_{n}$, i.e., $a_{n}$ and $b_{n}$, are divided by a constant to form the Fourier coefficients of $S C R_{n}$ as

$$
a_{n}^{\prime} \sim \mathrm{N}\left(\mu_{a_{n}}^{\prime}, \sigma_{c o f}^{\prime 2}\right), \quad b_{n}^{\prime} \sim \mathrm{N}\left(\mu_{b_{n}}^{\prime}, \sigma_{c o f}^{\prime 2}\right) \quad n \neq N
$$

where from linearity,

$$
\mu_{a_{n}}^{\prime}=\mu_{a_{n}} / E[C], \quad \mu_{b_{n}}^{\prime}=\mu_{b_{n}} / E[C], \quad \sigma_{c o f}^{\prime 2}=\sigma_{c o f}^{2} / E[C]^{2}
$$

For small delay SD values, $E[C]$ is simplified [16] to

$$
E[C] \approx \frac{2}{N \pi}\left|\frac{\sin \left(k N \pi / T_{r e f}\right) \sin \left(T_{s p e} N \pi / T_{r e f}\right)}{\sin \left(T_{s p e} \pi / T_{r e f}\right)}\right| \quad T_{s p e} \neq 0
$$

Therefore, it is concluded that $S C R_{n}$ is also a Ricean random variable with a PDF and mean defined in closed-from as

$$
\begin{aligned}
& p_{Z}(z)=\frac{z}{\sigma_{c o f}^{\prime 2}} \exp \left(-\frac{z^{2}+h^{\prime 2}}{2 \sigma_{c o f}^{\prime 2}}\right) I_{0}\left(\frac{h^{\prime} z}{\sigma_{c o f}^{\prime 2}}\right) \quad z \geq 0 \\
& E\left[S C R_{n}\right]=\sigma_{c o f}^{\prime} \sqrt{\frac{\pi}{2}} \times e^{\frac{-K^{\prime}}{2}} \times\left[\left(1+K^{\prime}\right) I_{0}\left(\frac{K^{\prime}}{2}\right)+K^{\prime} I_{1}\left(\frac{K^{\prime}}{2}\right)\right]
\end{aligned}
$$

where

$$
\begin{gathered}
h^{\prime}=\sqrt{\mu_{a_{n}}^{\prime 2}+\mu_{b_{n}}^{\prime 2}}=h / E[C] \\
K^{\prime}=h^{\prime 2} / 2 \sigma_{\text {cof }}^{\prime 2}=h^{2} / 2 \sigma_{\text {cof }}^{2}=K
\end{gathered}
$$

\section{VAlidation of the PRedictive Model}

This section evaluates the accuracy and robustness of the proposed prediction method of spur characterization of ECDLL-based synthesizers, over wide-range values of the system non-idealities. In order to do so, the predicted mean 


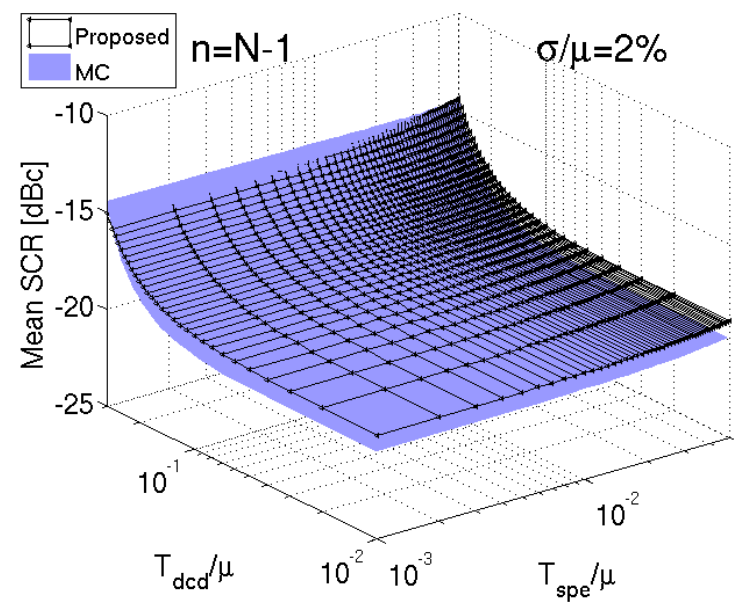

(a)

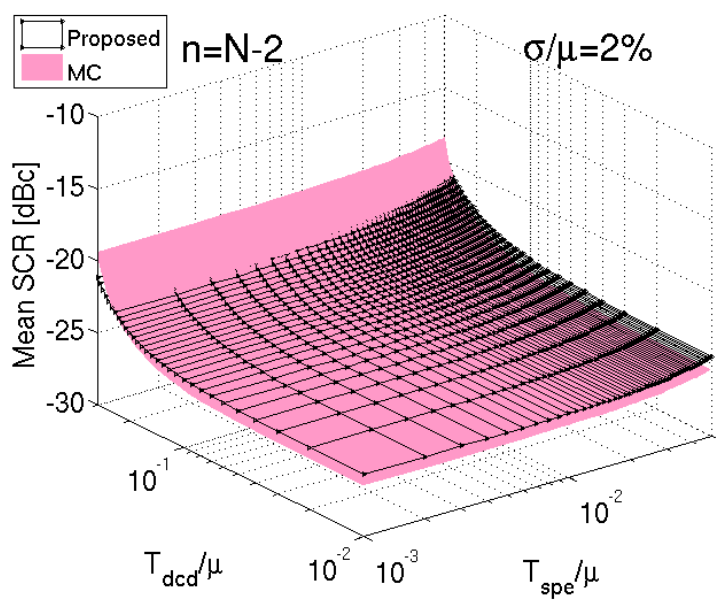

(b)

Fig. 9. MC simulation of the analytical model (9) versus the proposed predictive model (32) for mean SCR values as a function of normalized SPE and DCD, using normalized delay SD of $2 \%$ : (a) the adjacent, and (b) the alternate harmonic.

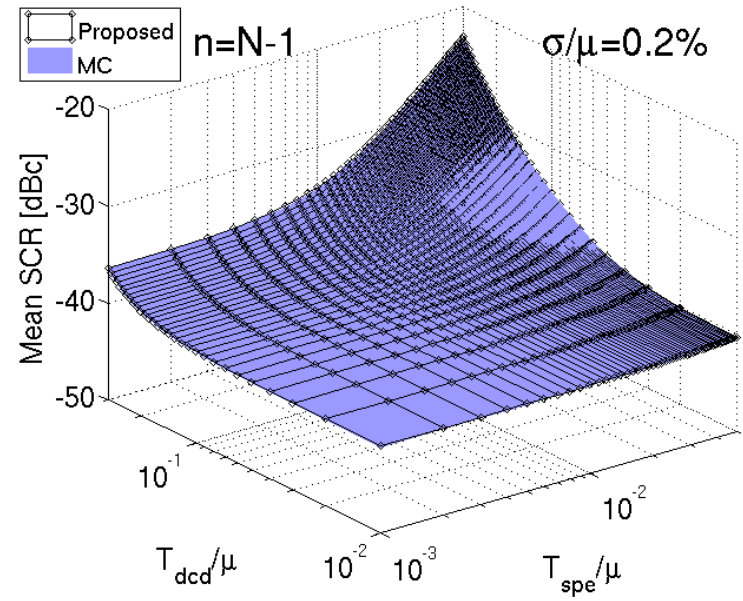

(a)

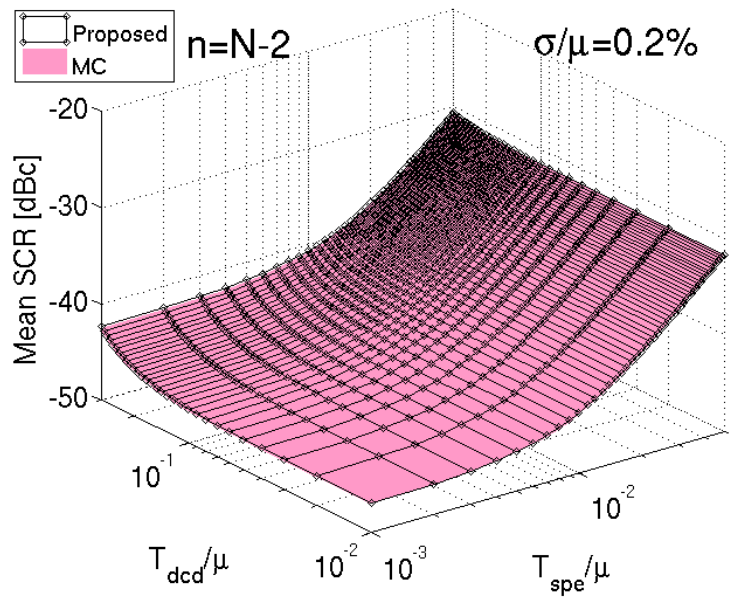

(b)

Fig. 10. MC simulation of the analytical model (9) versus the proposed predictive model (32) for mean SCR values as a function of normalized SPE and DCD, using normalized delay SD of $0.2 \%$ : (a) the adjacent, and (b) the alternate harmonic.

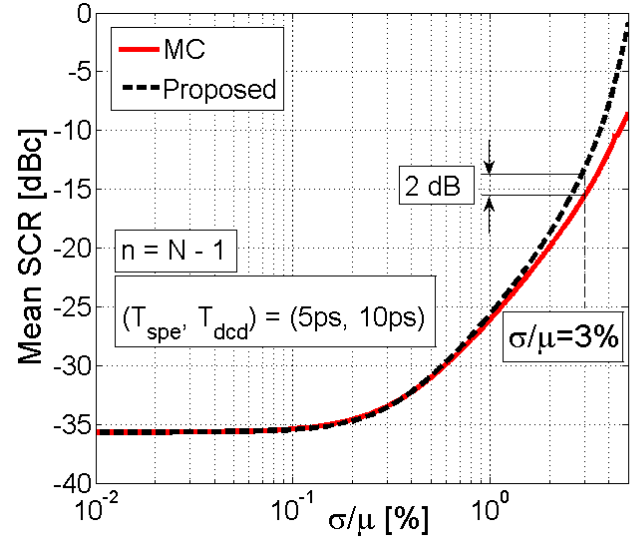

Fig. 11. MC simulation of the analytical model (9) versus the proposed predictive model for mean SCR values as a function of normalized delay SD.

SCR using the proposed model is compared with that of attained from the MC simulations of the analytical model (9). Furthermore, the accuracy of the proposed model is verified through MC simulations of the SCR for three different test cases, using a transistor-level ECDLL-based synthesizer model which is designed in a standard 65-nm CMOS technology.

\section{A. Behavioral Validation}

The mean SCR of the synthesizer as a function of SPE and DCD is illustrated in Fig. 9 and 10, using a normalized delay
SD of $2 \%$ and $0.2 \%$, respectively. Fig. 9(a) and 10(a) demonstrate the harmonic spur levels at $f_{s}=f_{c}-f_{\text {ref }}$, while Fig. 9 (b) and 10(b) illustrate the spur levels at $f_{s}=f_{c}-2 f_{\text {ref. }}$. The solid planes represent the MC simulation results of the analytical model of (9), whereas the solid lines with markers depict the predicted mean SCR provided by (32). It can be verified from Fig. 9 and 10 that the predicted results closely follow that of the MC simulations. So as to verify the accuracy of the proposed model against wide-range delay SD values, the mean SCR is plotted in Fig. 11 with respect to normalized delay SD which is swept from $0.01 \%$ to $5 \%$. It can be seen that the prediction results closely matches that of the $\mathrm{MC}$ simulation with an error of $0 \mathrm{~dB}$ for $\sigma / \mu=0.01 \%$ to $1 \%, 1 \mathrm{~dB}$ at $\sigma / \mu=2 \%$, and $2 \mathrm{~dB}$ at $\sigma / \mu=3 \%\left(S C R_{\text {mean }} \approx-15 \mathrm{dBc}\right)$. Note that to improve the prediction accuracy for very large delay SD values, higher order Taylor series can be employed to better approximate sine and cosine functions in (7) and (8), and hence, calculate the more exact model parameters, i.e., the mean and variance of $a_{n}$ and $b_{n}$ in (25), (26), and (27). Nevertheless, large delay SD values are in fact avoided due to the stringent requirements on the output spur levels in the context of frequency synthesis for wireless applications. Comparing the results in Fig. 9, 10, and 11 with those in Fig. 4,5 , and 6 , respectively, reveals the achieved improvements in the prediction robustness and implies that the proposed model 


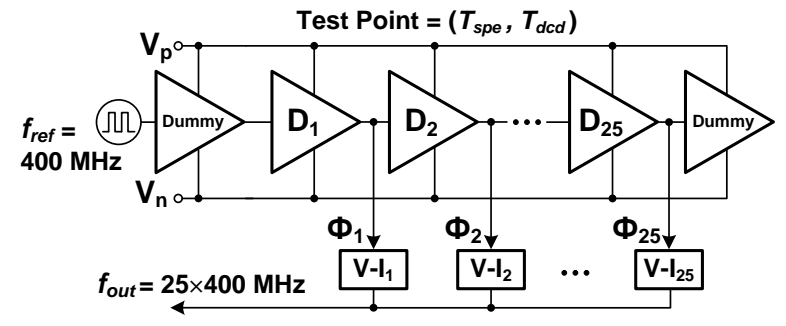

Fig. 12. Schematic of the simulated ECDLL synthesizer in open-loop regime.

can accurately characterize the spurious performance of ECDLLs for both the even and odd harmonic orders, over wide-range values of SPE, DCD, and delay SD.

\section{B. Transistor-Level Validation}

In the previous part, it was verified that the proposed predictive model closely matches the behavioral model of the ECDLL-based synthesizer for SCR estimation over wide-range values of the non-idealities. In this part, the validity and accuracy of the proposed model is investigated by comparing the predicted PDFs from the closed-form expression (31), with MC simulation histograms of the transistor-level model of the synthesizer, designed in a standard 65-nm CMOS process. Note that due to the large simulation time which is involved in MC simulations of the transistor-level design, it is not affordable to simulate the SCR for wide-range values of SPE and DCD. As a consequence, three different pairs of $\left(T_{s p e}, T_{d c d}\right)$ are instead selected for this experiment. Also, note that the reported SCR values in this part correspond to the largest adjacent and alternate harmonics, i.e., $\max \left(S C R_{N-1}, S C R_{N+1}\right)$ for the adjacent, and $\max \left(S C R_{N-2}, S C R_{N+2}\right)$ for the alternate harmonic. The simulated schematic of the ECDLL synthesizer is shown in Fig. 12, where 25 phase-shifted outputs of the VCDL are combined using 25 ideal stages of voltage-tocurrent (V-I) converters to generate the carrier at $f_{c}=10 \mathrm{GHz}$. Note that in practical implementations [8], [12], an LC-tank load can be used to enhance the ECDLL output impedance and also suppress the spur levels depending on its $Q$ factor [16]. So as to reduce the simulation time, the synthesizer is simulated in an open-loop regime. The effect of mismatch between up and down signals in PD/CP is modeled by manually introducing $T_{\text {spe }}$ in this open-loop schematic. Due to the open-loop operation, the actual SPE may slightly deviate from its designated value for each MC sample.

In order to obtain SCR for each MC sample, transient simulations are performed on the testbench of Fig. 12 for duration of $16.6 \mathrm{~ns}$, containing 5 cycles of the reference clock. Discrete Fourier transform (DFT) function is then applied to the synthesized output to calculate the corresponding magnitudes of the carrier and harmonic spurs. Simulations are performed using Cadence Spectre while accelerated parallel simulator (APS) is enabled. Transient noise option of the simulator is not enabled to speed up the MC simulations. However, since the noise will result in random jitter on the DLL output phases which is not identical from cycle to cycle, it does not produce a fixed-pattern periodicity. Therefore, it will give rise to the phase noise rather than the spur magnitude. The employed tolerance options of the simulator are reltol $=$ $10^{-6}$, vabstol $=10 \mathrm{nV}$, and iabstol $=100 \mathrm{fA}$. Furthermore, the simulations are carried out on a single $2.8-\mathrm{GHz}$ processor core, with 3.7 GB of memory. Considering the utilized configurations, the total simulation time regarding $10^{4} \mathrm{MC}$

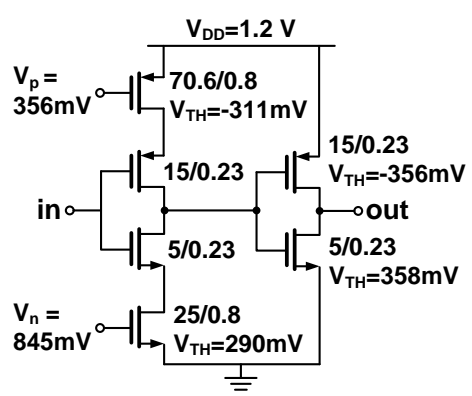

Fig. 13. Delay stage. Biasing corresponds to the case of zero SPE.

TABLE I

PREDICTION MODEL PARAMETERS AND THEIR RELATIONS WITH TECHNOLOGY, TRANSISTOR-LEVEL, AND SYSTEM PARAMETERS

\begin{tabular}{lccc}
\hline \hline $\begin{array}{c}\text { Prediction model } \\
\text { parameter }\end{array}$ & $\begin{array}{c}\text { Transistor-level } \\
\text { and technology } \\
\text { parameter }\end{array}$ & $\begin{array}{c}\text { System } \\
\text { parameter }\end{array}$ & Relation \\
\hline$\sigma_{r} / \mu_{r}, \sigma_{f} / \mu_{f}$ & $\begin{array}{c}\sigma_{l}, \sigma_{V T H}, V_{T H}, W L, V_{D D}, \\
V_{n}, V_{p}, A_{\beta}, A_{V T H}\end{array}$ & - & $(35)-(37)$ \\
$\lambda, \kappa_{c}, \kappa_{s}, \alpha_{m}, \beta_{m}$ & - & $T_{d c d}, T_{s p e}, n, N, T_{r e f}$ & $(15)-(17)$ \\
$\mu_{a n}, \mu_{b n}, \sigma_{c o f}$ & - & - & $(25)-(27)$ \\
$h, K$ & - & - & $(33),(34)$ \\
\hline \hline
\end{tabular}

samples is around $238 \mathrm{Ks}$ (23.8 s/sample). On the other hand, the predictions are carried out based on closed-from expressions where no simulation is involved. In order to plot the proposed SCR PDF of (31) for a given harmonic $n$, Table I summarizes how the prediction model parameters are related to the system, transistor-level, and technology parameters. As shown in Fig. 13, a current-starved delay stage with an output inverter buffer is utilized and carefully designed to provide a mean delay of $\mu=\mu_{r} \approx \mu_{f} \approx 100$ ps. Employing the mismatch equations for MOS devices [17], the value of the normalized stage-delay SD for a current-starved delay stage of Fig. 13 is derived [13] as

$$
\frac{\sigma}{\mu}=\sqrt{\left(\frac{\sigma_{I}}{I}\right)^{2}+\left(\frac{\sigma_{V_{T H}}}{V_{D D}-V_{T H}}\right)^{2}}
$$

where $\sigma_{I}$ and $\sigma_{V T H}$ are the current and voltage-threshold SDs,

$$
\begin{gathered}
\frac{\sigma_{I}}{I}=\frac{A_{\beta}}{\sqrt{W L_{C S}}}+\frac{2 A_{V_{T H}}}{\left(V_{G S}-V_{T H}\right) \sqrt{W L_{C S}}} \\
\sigma_{V_{T H}}=\frac{A_{V_{T H}}}{\sqrt{W L_{B}}}
\end{gathered}
$$

Also, $W L_{C S}$ and $W L_{B}$ are the areas of the current-starved and buffer transistors, and $A_{\beta}$ and $A_{V T H}$ are the technologydependent constants. With the transistor sizing and voltage biasing shown in Fig. 13, and the technology constants of the target standard 65-nm CMOS process, the delay SD values of $\sigma_{r} / \mu_{r} \approx 0.27 \%$ and $\sigma_{f} / \mu_{f} \approx 0.29 \%$ are obtained. So as to observe the accuracy of the predictive model when the delay mismatch dominates SPE and DCD values, a SPE-DCD pair of $(0,0)$ is selected as the first test case. It can be perceived from the SCR distributions in Fig. 14, that both the PDFs of the proposed predictive model (solid line with markers) given by (31), and the approximation model of [16] (dashed line) closely match the histogram of the transistor-level MC simulations (bar chart), for either of the harmonics. It can also be verified that for this test case where the delay SD absorbs SPE and DCD values, the mean SCR of the adjacent harmonic, shown in Fig. 14(a), is larger than that of the alternate 


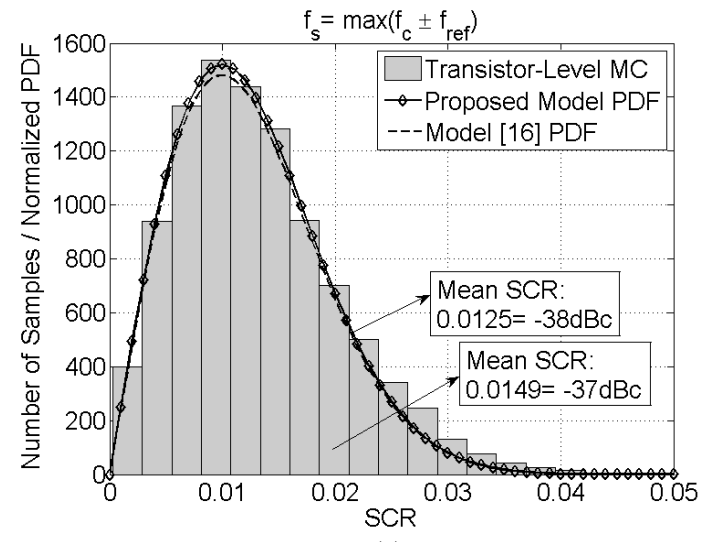

(a)

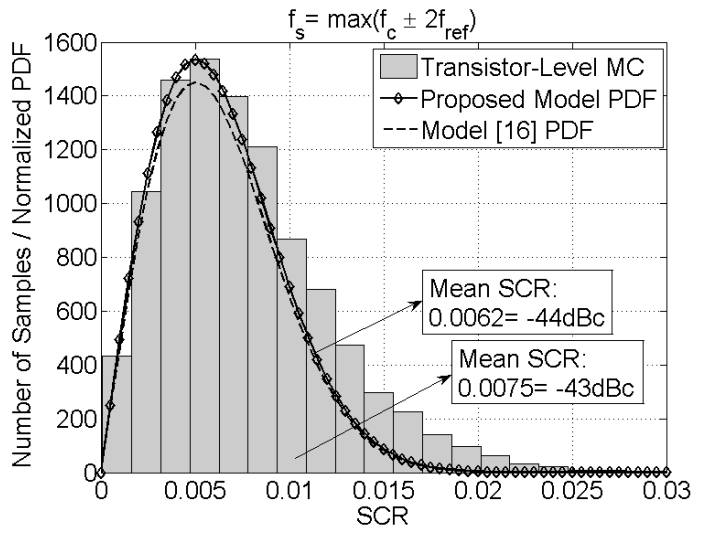

(b)

Fig. 14. Predicted PDF versus transistor-level MC histogram of SCR with $\left(T_{s p e}, T_{d c d}\right)=(0,0)$ : (a) the largest adjacent, and (b) the largest alternate spur.

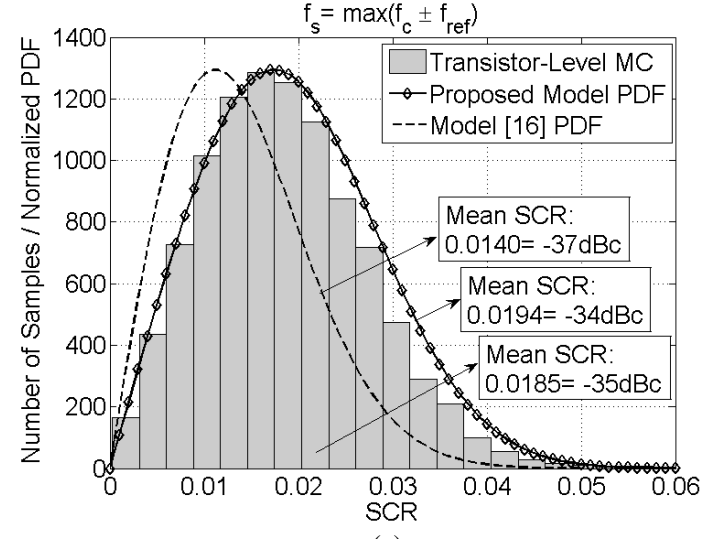

(a)

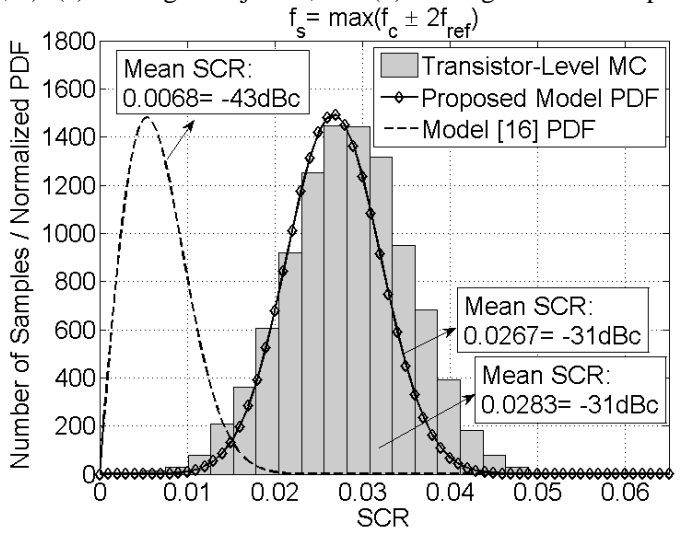

(b)

Fig. 15. Predicted PDF versus transistor-level MC histogram of SCR with $\left(T_{s p e}, T_{d c d}\right)=(5 \mathrm{ps}, 9.3 \mathrm{ps})$ : (a) the largest adjacent, and (b) the largest alternate spur.

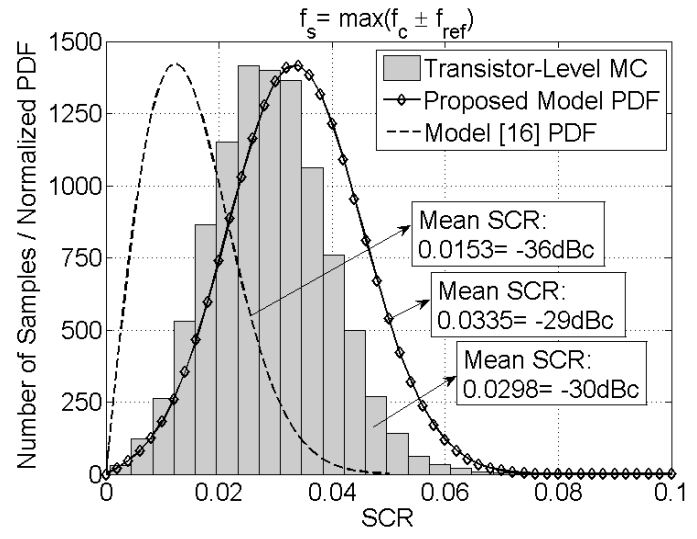

(a)

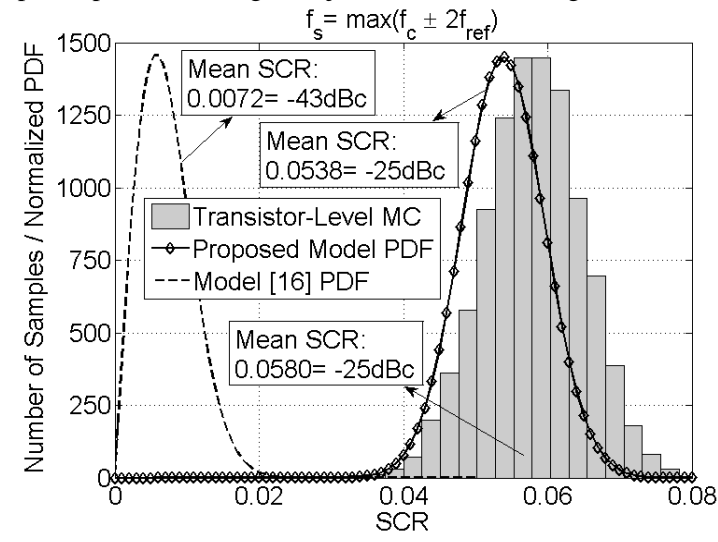

(b)

Fig. 16. Predicted PDF versus transistor-level MC histogram of SCR with $\left(T_{s p e}, T_{d c d}\right)=(10 \mathrm{ps}, 9.3 \mathrm{ps})$ : (a) the largest adjacent, and (b) the largest alternate spur.

harmonic plotted in Fig. 14(b). The second test is performed using the non-ideality pair (5ps, 9.3ps). Note that the required $T_{s p e}=5 \mathrm{ps}$ is introduced to both the rising and falling-edge delays, by modifying the bias voltages to $V_{n}=851.6 \mathrm{mV}$ and $V_{p}=347.3 \mathrm{mV}$ in Fig. 13. Moreover, the value of DCD is initially set to $T_{d c d}=10 \mathrm{ps}$ by changing the pulsewidth of the square wave input clock source in the schematic testbench of Fig. 12. The effective DCD value however, is about $9.3 \mathrm{ps,}$ according to the transient simulation of the waveforms. A similar SPE-DCD pair has been utilized as the input to the PDF expressions of the predictive models and the comparison results are depicted in Fig. 15. It can be seen that the SCR of the alternate harmonic dominates that of the adjacent harmonic by $4 \mathrm{~dB}$. It can also be perceived that the model of [16] exhibits a prediction error of $2 \mathrm{~dB}$ and $12 \mathrm{~dB}$, for the adjacent and alternate harmonic spurs, respectively, while the proposed model predicts the mean SCR accurately for both harmonics. As the last experiment and to evaluate the accuracy of the model when the delay mismatch is absorbed by relatively large SPE and DCD values, a test pair of (10ps, 9.3ps) is employed. To provide the required $T_{s p e}=10 \mathrm{ps}$, the bias voltages of the current-starved delay elements are changed to $V_{n}=858.7 \mathrm{mV}$ and $V_{p}=338.1 \mathrm{mV}$. It can be noticed from Fig. 16 that in this case, the PDF of the proposed model can follow the histogram of the transistor-level MC simulations for both the adjacent and alternate harmonic spurs, while the prediction error of [16] is magnified to $6 \mathrm{~dB}$ and $18 \mathrm{~dB}$.

\section{CONCLUSION}

Evaluation of spurious performance in ECDLL-based 
frequency synthesizers demands exhaustive statistical simulations using MC method which significantly slow down the iterative design procedure of such synthesizers. Based on the derived closed-form expressions for the PDF and mean of the spur magnitudes, this paper introduces a generic predictive model for characterizing the spurious performance of ECDLLbased synthesizers. The important characteristic of the proposed model is that its prediction accuracy is independent of the design parameters and the system non-idealities. Hence, the model is comparable to MC method in terms of accuracy and robustness, whereas it alleviates the need for exhaustive statistical simulations. The accuracy of the model has been investigated through MC simulations of the behavioral model of the synthesizer, against wide-range values of SPE, DCD, and stage-delay SD. Moreover, the validity of the model has been further inspected by performing MC simulations on a transistor-level model of the synthesizer which is designed in a standard 65-nm CMOS process. Comparison of the simulated and estimated results verifies that the predictive model is generic, and hence, can be considered as a reliable replacement to $\mathrm{MC}$ method for characterizing the spurious performance of ECDLL-based frequency synthesizers.

\section{REFERENCES}

[1] G. Marucci, et al., "A 1.7 GHz MDLL-based fractional-N frequency synthesizer with $1.4 \mathrm{ps}$ RMS integrated jitter and $3 \mathrm{~mW}$ power using $1 \mathrm{~b}$ TDC," in Proc. IEEE Int. Solid-State Circuits Conf., Dig. Tech. Papers, Feb. 2014, pp. 360-361.

[2] A. Elshazly et al., "A $1.5 \mathrm{GHz} 890 \mathrm{uW}$ digital MDLL with $400 \mathrm{fsrms}$ integrated jitter, $-55.6 \mathrm{dBc}$ reference spur and $20 \mathrm{fs} / \mathrm{mV}$ supply-noise sensitivity using 1b TDC," in Proc. IEEE Int. Solid-State Circuits Conf., Dig. Tech. Papers, Feb. 2012, pp. 242-244.

[3] T. Ali, et al., "A 4.6GHz MDLL with $-46 \mathrm{dBc}$ reference spur and aperture position tuning," in Proc. IEEE Int. Solid-State Circuits Conf. Dig. Tech. Papers, Feb. 2011, pp. 466-467.

[4] B. M. Helal, et al., "A highly digital MDLL-based clock multiplier that leverages a self-scrambling time-to-digital converter to achieve subpicosecond jitter performance," IEEE J. Solid-State Circuits, vol. 43, no. 4, pp. 855-863, Apr. 2008.

[5] S. Gierkink, "Low-spur, low-phase-noise clock multiplier based on a combination of PLL and recirculating DLL with dual-pulse ring oscillator and self-correcting charge pump," IEEE J. Solid-State Circuits, vol. 43, no. 12, pp. 2967-2976, Dec. 2008.

[6] P. C. Maulik and D. A. Mercer, "A DLL-based programmable clock multiplier in $0.18 \mu \mathrm{m}$ CMOS with $-70 \mathrm{dBc}$ reference spur," IEEE J. Solid-State Circuits, vol. 42, no. 8, pp. 1642-1648, Aug. 2007.

[7] G. Marzin, et al., "A spur cancellation technique for MDLL-based frequency synthesizers," in Proc. IEEE Int. Symposium Circuits Syst., May 2013, pp. 165-168.

[8] G. Chien and P. R. Gray, "A 900-MHz local oscillator using a DLLbased frequency multiplier technique for PCS applications," IEEE $J$. Solid State Circuits, vol. 35, no. 12, pp. 1996-1999, Dec. 2000.

[9] J. Zhuang, Q. Du, and T. Kwasniewski, "A -107 dBc $10 \mathrm{kHz}$ carrier offset 2-GHz DLL-based frequency synthesizer," in Proc. IEEE Custom Integr. Circuits Conf., Sep. 2003, pp. 301-304.

[10] J. Zhuang, Q. Du and T. Kwasniewski, "Noise, spur characteristics and in-lock error reduction of DLL-based frequency synthesizers," in Proc. Int. Conf. Commun. Circuits Syst., Jun. 2004, pp. 1443-1446.

[11] A. Ojani, B. Mesgarzadeh, and A. Alvandpour, "A DLL-based injection-locked frequency synthesizer for WiMedia UWB," in Proc. IEEE Int. Symposium Circuits Syst., May 2012, pp. 2027-2030.

[12] T. C. Lee and K. J. Hsiao, "The design and analysis of a DLL-based frequency synthesizer for UWB application," IEEE J. Solid State Circuits, vol. 41, no. 6, pp. 1245-1252, Jun. 2006.
[13] O. Casha et al., "Analysis of the spur characteristics of edge-combining DLL-based frequency multipliers," IEEE Trans. Circuits Syst. II: Exp. Briefs, vol. 56, no. 2, pp. 132-136, Feb. 2009.

[14] N. Khan, M. Hossain, K.L.E. Law, "A low power frequency synthesizer for $60-\mathrm{GHz}$ wireless personal area networks," IEEE Trans. Circuits Syst. II: Exp. Briefs, vol.58, no.10, pp. 622-626, Oct. 2011.

[15] H. J. Ng et al., "A DLL-supported, low phase noise fractional-N PLL with a wideband $\mathrm{VCO}$ and a highly linear frequency ramp generator for FMCW radars," IEEE Trans. Circuits Syst. I: Reg. Papers, vol. 60, no. 12, pp. 3289-3302, Dec. 2013.

[16] A. Ojani, B. Mesgarzadeh, and A. Alvandpour, "Modeling and analysis of harmonic spurs in DLL-based frequency synthesizers," IEEE Trans. Circuits Syst. I: Reg. Papers, vol. PP, no. 99, pp. 1-10, Jun. 2014.

[17] M. J. M. Pelgrom, A. C. J. Duinmaijer, and A. P. G. Welbers, "Matching properties of MOS transistors," IEEE J. Solid-State Circuits, vol. 24, no. 10, pp. 1433-1440, Oct. 1989.

[18] T. P. Gia, N. Turkkan, and E. Marchand, "Density of the ratio of two normal random variables and applications," Commun. Stat., Theory Methods, vol. 35, no. 7-9, pp. 1569-1591, Sep. 2006.

[19] J.G. Proakis, and M. Salehi, Digital Communications, $5^{\text {nd }}$ Edition, McGraw Hill, 2008.

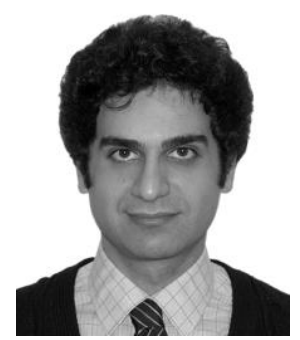

Amin Ojani (S'10) received the M.Sc. degree in electrical engineering from Linköping University, Linköping, Sweden, in 2008. He is currently working towards the Ph.D. degree at Linköping University.

Between 2008 and 2009, he was with Ericsson $\mathrm{AB}$, Lund, Sweden, where he worked on clock generation and distribution for Ericsson's mobile platforms. His research interests include phaselocked systems and RF synthesizers.

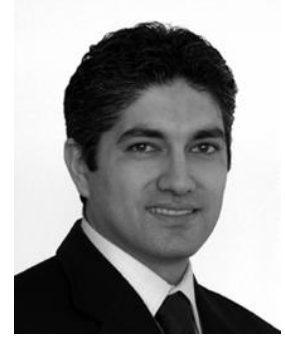

Behzad Mesgarzadeh (S'02-M'09) received the B.Sc. degree in electrical engineering from Sharif University of Technology, Tehran, Iran, in 2000, and the M.Sc. and Ph.D. degrees in electrical engineering from Linköping University, Linköping, Sweden, in 2004 and 2008, respectively.

$\mathrm{He}$ is currently an Assistant Professor at the Department of Electrical Engineering, Linköping University. His research interests include lowpower clocking techniques, clock generators and frequency synthesizers, and high-data-rate wireless communication systems.

Dr. Mesgarzadeh was the recipient of the 50th IEEE Midwest Symposium on Circuits and Systems Best Student Paper Award in 2007.

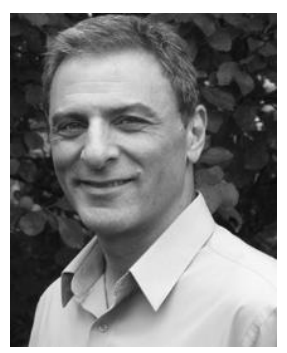

Atila Alvandpour (M'99-SM'04) received the M.S. and Ph.D. degrees from Linköping University, Sweden, in 1995 and 1999, respectively.

From 1999 to 2003, he was a senior research scientist with Circuit Research Lab, Intel Corporation. In 2003, he joined the department of Electrical Engineering, Linköping University, as a Professor of VLSI design. Since 2004, he is the head of Electronic Devices division. His research interests include various issues in design of integrated circuits and systems in advanced nanoscale technologies, with a special focus on efficient data converters, wireless transceiver front-ends, onchip clock generators and synthesizers, sensor interface electronics, lowpower/high-performance digital circuits and memories, and chip design techniques. He has published more than 100 papers in international journals and conferences, and holds 24 U.S. patents.

Prof. Alvandpour is a senior member of IEEE, and has served on many technical program committees of IEEE and other international conferences, including the IEEE Solid-State Circuits Conference (ISSCC), and European Solid-State Circuits Conference (ESSCIRC). He has also served as guest editor for IEEE JOURNAL OF SOLID-STATE CIRCUITS. 